\title{
Savoirs en miroir
}

\section{Christian Jacob}

\section{(2) OpenEdition \\ Journals}

Édition électronique

URL : https://journals.openedition.org/rbnu/2673

DOI : $10.4000 /$ rbnu. 2673

ISSN : 2679-6104

\section{Éditeur}

Bibliothèque nationale et universitaire de Strasbourg

\section{Édition imprimée}

Date de publication : 1 novembre 2012

Pagination : 8-17

ISSN : 2109-2761

\section{Référence électronique}

Christian Jacob, "Savoirs en miroir», La Revue de la BNU [En ligne], 6 | 2012, mis en ligne le 01 novembre 2012, consulté le 09 août 2021. URL : http://journals.openedition.org/rbnu/2673 ; DOI https://doi.org/10.4000/rbnu.2673

\section{(c) (i) (3)(2)}

La Revue de la BNU est mise à disposition selon les termes de la Licence Creative Commons Attribution - Pas d'Utilisation Commerciale - Partage dans les Mêmes Conditions 4.0 International. 


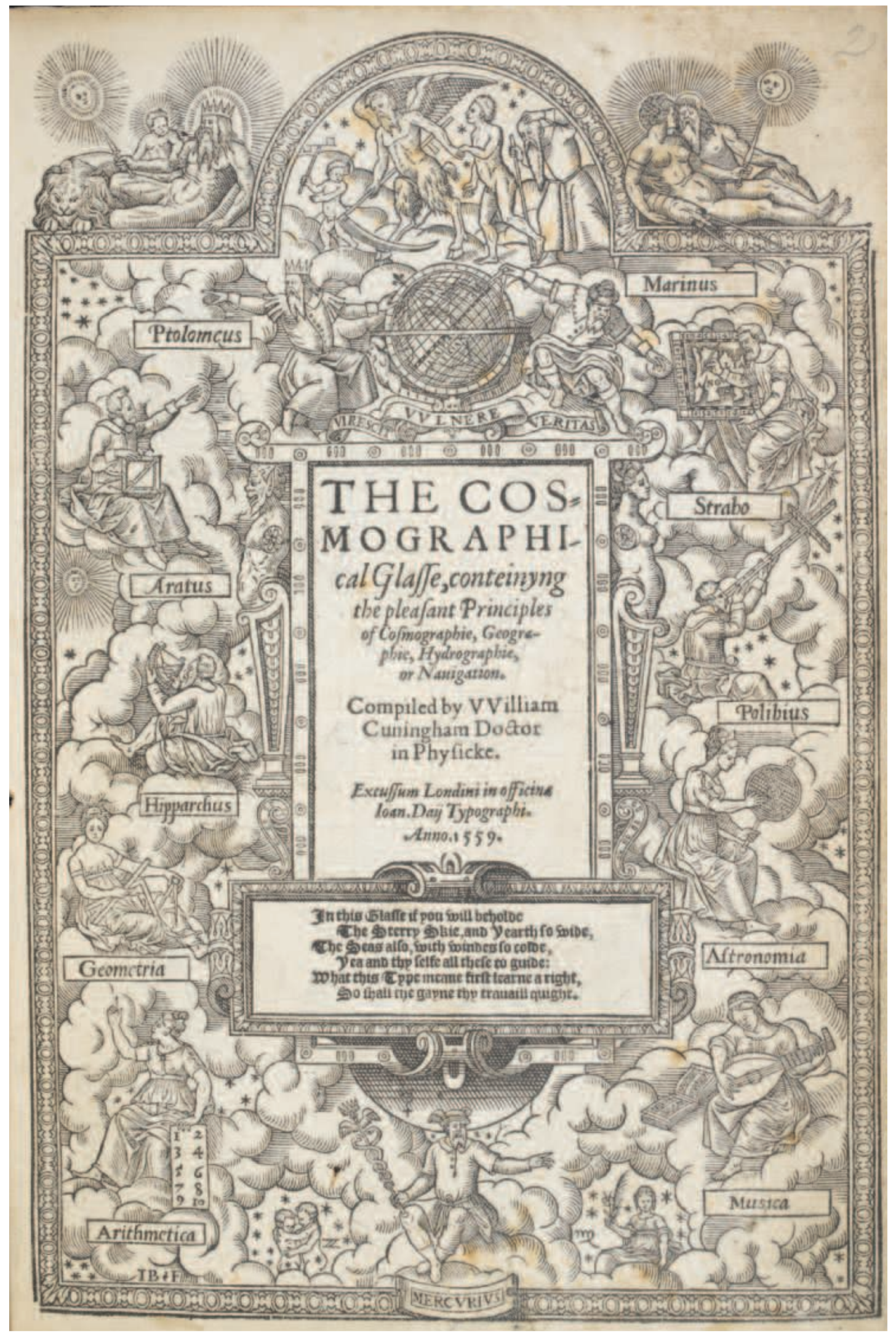

Page de titre du Cosmographical Glasse de William Cuningham (1559 - coll. British Library) 


\section{SAVOIRS EN MIROIR}

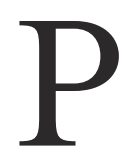

iège de l'apparence, qui ne produit qu'un leurre visuel et ontologique? Ou interface technique et magique révélant immédiatement le monde dans sa réalité et sa vérité ? Dans les cultures occidentale et orientale, le miroir est un instrument de connaissance, révélant au regard humain les êtres et les choses, toutes les dimensions du visible et de l'invisible, à travers même les jeux d'illusions qu'il génère ${ }^{1}$. Ses riches connotations métaphoriques sous-tendent son emploi dans les titres d'ouvrages du Moyen Age et de la Renaissance ${ }^{2}$ : Miroir, Mirror, Speculum, Specchio, Spieghel, il désigne des textes à finalité éthique et instructive, dont il souligne le pouvoir d'édification et d'exemplarité morale. La littérature anglaise abonde ainsi en Specula pour les enfants, les vierges, les moines et les pêcheurs, les laïcs et les parieurs, les célibataires ${ }^{3}$. Reflétant fidèlement la réalité humaine, le miroir en révèle les défauts et les ridicules par son pouvoir grossissant : il est alors l'instrument de la satire et de la dérision. Montrant le monde tel qu'il est sur une surface réduite et offerte au regard, le miroir est enfin instrument de connaissance, encyclopédique ou spécialisée, théorique ou pratique. La métaphore du miroir apparaît déjà dans le De imagine mundi d'Honoré d'Autun $\left(12^{\mathrm{e}} \mathrm{s}\right.$.). L'auteur justifie ainsi son titre : " parce que l'on peut voir [dans ce livre] la disposition de la Terre tout entière, comme dans un miroir, quasi in speculo conspiciatur $"{ }^{4}$. Cette encyclopédie sera rééditée sous le titre de Speculum mundi, témoignant, comme le note Herbert Grabes, du passage de l'image (contenu de la vision) à la réflexion (la modalité de production du savoir) ${ }^{5}$. Le Speculum quadruplex ou Speculum majus de Vincent de Beauvais constitue l'encyclopédie médiévale la plus complète. La tradition se perpétue durant la Renaissance, avec le Mirrour of the world de Caxton et le Speculum mundi de John Swan. Le livre encyclopédique partage avec le miroir du Moyen Age et du début de la Renaissance, convexe dans la plupart des cas, les mêmes propriétés : " réflexion et réduction, (re-) présentation et compression $"{ }^{6}$. Un livre doit être aussi maniable qu'un miroir, et l'information aussi directement accessible que par un regard sur une surface réfléchissante. Speculum est ainsi un titre privilégié pour des ouvrages à vocation didactique, qui exposent sous une forme condensée l'essentiel du savoir dans un champ déterminé. La très grande majorité des "Miroirs " didactiques se limitent en effet à un savoir technique ou à une discipline, la grammaire, l'alchimie, l'histoire, la médecine ${ }^{7}$. La géographie est l'une d'elles.

Les traités géographiques de la Renaissance dont le titre renvoie à la symbolique du miroir entendent tout d'abord signifier leur vocation didactique, voire encyclopédique, et leur appartenance à un genre littéraire bien établi. Mais la présence des cartes redonne au miroir une signification visuelle forte, celle d'une interface technique ouvrant un espace de vision inédit sur une réalité inaccessible. 
Les significations métaphoriques du miroir sont souvent explicitées par les préfaces, voire par le frontispice des ouvrages eux-mêmes. La page de titre emblématique a pour fonction de " communiquer des idées complexes au moyen d'images visuelles " ${ }^{8}$ et elle impose souvent à son lecteur l'effort du déchiffrement, voire le plaisir de décoder l'image, si l'on possède l'érudition nécessaire. Elle est en outre un lieu privilégié où l'auteur traduit visuellement la nature et l'intention de son œuvre' .

Tel est le cas, par exemple, de l'ouvrage de William Cuningham, The Cosmographical Glasse, publié à Londres en $1559^{10}$. Il s'agit d'un traité relativement bref (un peu plus de deux cents pages), qui expose les rudiments du savoir cosmographique sous une forme résumée et accessible (un dialogue). Le titre complet de l'ouvrage, en effet, précise : "The Cosmographical Glasse, conteinyng the pleasant Principles of Cosmographie, Geographie, Hydrographie, or Navigation ". Cuningham s'est inspiré des traités d'Apian et d'Oronce Finé, et il est l'auteur du premier manuel de cosmographie en langue anglaise. A défaut d'être une œuvre originale, The Cosmographical Glasse a contribué à diffuser l'intérêt pour la géographie et les cartes dans la société anglaise.

Le frontispice offre une construction graphique complexe $^{11}$ : au centre, le carton de titre surmontant un cartouche qui porte cette inscription :

" In this Glasse if you will beholde

The Sterry skie, and Yearth so wide,

The Seas also, with windes so colde,

Yea and thy selfe all these to guide:

What this Type meane first learne a right,

So shall the gayne thy travaill quight ".

Tout autour se déploient des scènes juxtaposées, où des personnages, assis au milieu des nuées, sont absorbés dans différentes activités d'observation et de calcul, combinant gestes déictiques et regards attentifs. Ces personnages sont identifiés par leur nom. Ils se regroupent en deux grandes catégories. Tout d'abord, les allégories des arts et des sciences (des figures féminines) : Geometria, Arithmetica, Musica, Astronomia ; nous trouvons ici réunies les disciplines du " quadrivium " médiéval. Ensuite, les grands géographes et astronomes de l'Antiquité : Hipparque, Aratos, Strabon, Polybe. Ces différentes figures des- sinent, mesurent, calculent, munies des instruments de la géométrie, de l'astronomie et de la cartographie. Dans l'angle supérieur droit, Strabon met la dernière main à une carte de l'Angleterre, orientée l'ouest en haut et tracée sur un espace orthonormé, gradué en degrés de longitude et de latitude : un exemple parfait de planche chorographique à la manière de Ptolémée. C'est d'ailleurs Ptolémée, à côté de son collègue et prédécesseur Marin de Tyr, qui donne la clé de l'ensemble $\mathrm{du}$ dessin, au centre du frontispice et au-dessus du cartouche de titre. Les deux savants entourent un globe terrestre. Le réseau des méridiens et des parallèles laisse transparaître une carte à peine esquissée, où on lit les noms Asia et Africa. Ptolémée a le doigt pointé vers les étoiles, tandis que Marin mesure au compas un écart de latitude sur le globe, de la main droite, et tend la main gauche vers la carte de l'Angleterre, que dessine Strabon. La sphère terrestre est la médiation qui permet de passer du ciel à l'Angleterre. Elle est aussi l'artefact global dont la genèse et les composantes partielles sont illustrées par l'ensemble des figures qui l'entourent : savoirs scientifiques et gestes techniques, qui mettent en œuvre la panoplie des instruments d'observation de la Renaissance : compas, sextants, etc. Cette représentation complexe est ainsi une mise en abyme du miroir et de ses pouvoirs synoptiques : le frontispice reflète la cosmographie dans la diversité de ses disciplines et la continuité de son histoire, depuis l'Antiquité. Ces différentes facettes de la cosmographie se résument elles-mêmes dans le globe terrestre. La surface graphique du frontispice promet la complétude du savoir comme sa miniaturisation synoptique.

D'autres éléments, empruntés au symbolisme zodiacal, viennent préciser la signification de l'ensemble. En bas, au centre de la page, Mercure est flanqué des Gémeaux et de la Vierge qui sont ses deux " maisons $"{ }^{12}$. Il est significatif que Mercure soit également entouré d'Arithmetica et de Musica. Au sommet du frontispice, et au-dessus du globe terrestre sur lequel travaillent Ptolémée et Marin, se trouve représenté Saturne sous la forme d'un vieillard ailé aux jambes de bouc, muni d'une faux, symbolisant ses pouvoirs destructeurs ${ }^{13}$. Autour de lui, l'enfant, l'adulte, qu'il tient par la main et, derrière, le vieillard symbolisent les trois âges de l'être humain et l'évolution qui le conduit de la naissance à la mort. A droite et à gauche, et endehors du cadre du frontispice, se trouve une repré- 


\section{Cuningham évoque le combat entre l'ignorance et la connaissance : en aspirant à cette dernière, l'homme s'arrache à la bestialité pour se rapprocher de Dieu.}

sentation du soleil et de la lune, éclairant deux figures allongées, un vieillard et une femme, dans la position traditionnellement reconnue aux allégories des fleuves. Le soleil et la lune sont accompagnés par un lion et une écrevisse (le Cancer) qui les symbolisent. Peut-être avons-nous ici la représentation des deux hémisphères de la Terre dont l'un est plongé dans l'obscurité et l'autre éclairé par le soleil ${ }^{14}$. L'association du globe terrestre et de Saturne n'est pas arbitraire : on connaît des représentations où Saturne pointe un compas vers la surface du globe, montrant ainsi l'emprise du temps sur la Terre ${ }^{15}$. Et ce lien entre le temps et la cosmographie, s'il est renforcé par la présence de l'historien Polybe, est aussi manifesté par l'étrange rassemblement des grands noms de l'astronomie et de la géographie hellénistiques et impériales, du $3^{\mathrm{e}} \mathrm{s}$. avant J.-C. au $2^{\mathrm{e}} \mathrm{s}$. après.

Dans sa dédicace à Lord Robert Duddeley, Cuningham évoque le combat entre l'ignorance et la connaissance : en aspirant à cette dernière, l'homme s'arrache à la bestialité pour se rapprocher de Dieu. C'est pour lutter contre l'ignorance que l'auteur a conçu " ce miroir, ou Verre Cosmographique, dans lequel les hommes peuvent regarder attentivement non pas un ou deux personnages, mais le ciel avec ses planètes et ses étoiles, la Terre avec ses belles régions, et les Mers, avec leurs merveilleuses marées $"{ }^{16}$. La métaphore du miroir ressurgit dans le cours du texte, lorsque l'élève, Spoudaeus, qui vient d'apprendre de son maître Philonicus les principes de la cartographie, s'exclame ${ }^{17}$ : " Ainsi en respectant l'ordre que vous m'avez appris, je peux en quelque sorte selon mon bon plaisir, dessiner une carte pour l'Espagne, la France, l'Allemagne, l'Italie, la Grèce, ou toute autre région particulière : oui, dans une maison chaude et agréable, sans les périls des mers déchaînées, le danger des ennemis, la perte de temps, la dépense de l'argent, les blessures du corps ou les angoisses de l'esprit. Oh quel précieux joyau que voici, on peut l'appeler à juste titre un Verre Cosmographique, dans lequel nous pouvons observer la diversité des pays, la nature des peuples, et les formes innombrables des Bêtes, des Foules, des Poissons, des Arbres, des Fruits, des Fleuves et des Métaux ".

Je prendrai un second exemple dans la production éditoriale des Pays-Bas. L'imprimeur et graveur Gérard de Jode (1509-1591) publie en 1578 un Speculum Orbis Terrarum, qui constitue l'un des premiers atlas modernes, aux côtés du Theatrum Orbis Terrarum d'Ortelius en 1570 et de l'Atlas de Mercator $^{18}$. Particulièrement intéressant est le titre choisi par de Jode pour différencier son ouvrage du Theatrum d'Ortelius et le concurrencer - ce qu'Ortelius avait du reste bien compris, puisqu'il a sans doute contribué à faire retarder la publication du nouvel atlas de 
plusieurs années ${ }^{19}$. Contrairement à l'ouvrage de Cuningham, le Speculum n'est pas un manuel de cosmographie générale, mais un recueil de 90 cartes, distribuées en deux parties, redessinées et uniformisées de façon à former un tout cohérent ${ }^{20}$. Cornelis de Jode succède à son père et réalise la seconde édition du Speculum, revue et augmentée, car composée de 109 cartes, et publiée à Anvers en 1593. Pour la circonstance, de nouvelles pages de titre ont été gravées : la principale représente un cheval, un chameau, un lion et un lama, symbolisant les quatre continents, l'Europe, l'Afrique, l'Asie et l'Amérique.

Le frontispice de la première partie offre une composition graphique complexe. La gravure n'est pas signée. Mais la sophistication du programme décoratif, et sa nature emblématique, suggèrent que Gérard de Jode en a été le concepteur, voire le graveur. Au centre de la page, un miroir ovoïde, entouré d'un cadre découpé en douze cases où sont représentés les signes du zodiaque, porte l'inscription "Speculum Orbis Terrarum ". On décèle sous les lettres un tracé au trait très schématique, à peine visible, figurant les formes de l'Afrique, de l'Arabie, de l'Asie mineure et de l'Europe. Cette surface légèrement convexe est un espace de vision et de contemplation : entourée de huit têtes de chérubins - les vents - elle voit apparaître le contour de la Terre sans les artifices de la cartographie - lignes, grille, inscriptions - mais avec la surimposition d'un titre qui identifie à la fois le contenu et le support de l'image. Le statut de cette vision est certainement qualifié par les hiéroglyphes gravés sur les deux obélisques qui encadrent le miroir et au sommet desquels se trouvent le soleil et la lune. Le frontispice du Speculum utilise une imagerie égyptienne caractéristique de la Renaissance, qui se perpétuera au $17^{\mathrm{e}}$ siècle ${ }^{21}$. Les pyramides et les hiéroglyphes sont les emblèmes d'une sagesse cachée, où se mêlent la tradition de l'hermétisme gréco-égyptien et son interprétation par les néoplatoniciens de la Renaissance ${ }^{22}$. Depuis Plotin ${ }^{23}$, et grâce à Marsile Ficin qui fut son traducteur, les hiéroglyphes sont considérés comme l'expression ultime de la vérité, car ils incorporent les concepts sous une forme visuelle davantage qu'ils ne les figurent. Les hiéroglyphes de de Jode dérivent très probablement des Hieroglyphica de Horapollo, manuscrit grec importé à Florence au début du $15^{\mathrm{e}}$ siècle, qui offrit une clé de déchiffrement allégorique pour toute la Renaissance. Ainsi l'œil désigne Dieu qui voit et sait tout, le serpent qui se mord la queue le temps, etc. Au pied de ces obélisques se trouvent un lion et une écrevisse (équivalent figuratif du Cancer zodiacal) : ils sont respectivement les attributs du soleil et de la lune. Les hiéroglyphes donnent au livre ainsi introduit le statut d'un savoir révélé et mystérieux, et on pourrait considérer aussi que cette profusion de signes, de symboles zodiacaux et de figures allégoriques déploie un horizon de déchiffrement et d'efforts herméneutiques autour de l'évidence visuelle offerte par le miroir central, où les contours $\mathrm{du}$ monde apparaissent mystérieusement au regard. Les formes de la Terre surgissent en effet comme dans un au-delà de la vision sensorielle, comme l'expression d'une réalité idéelle, se dévoilant grâce à une expérience inteldes sagesses.

lectuelle qui s'apparente à la plus haute

Au pied de ce dispositif architectural se trouvent deux anges. Celui de gauche, sous l'inscription " Hydrographia ", tient un compas marin et une règle. Celui de droite, sous l'inscription " Horologiographia ", tient un instrument de mesure. A ses pieds se trouve un sablier, pour la mesure du temps. Au centre, se trouve assise une femme drapée, "Geographia ", tenant dans la main droite un compas ouvert et dans la main gauche, une pyramide. Sa tête est surmontée d'une tour. Elle est assise sur une table basse (" tabula " la carte géographique ?), dont les deux montants verticaux portent les inscriptions "Asia " et "Africa " et le montant horizontal l'inscription " Europa ". La partie inférieure de l'image réunit les instruments techniques de la cartographie, ensemble de média- 


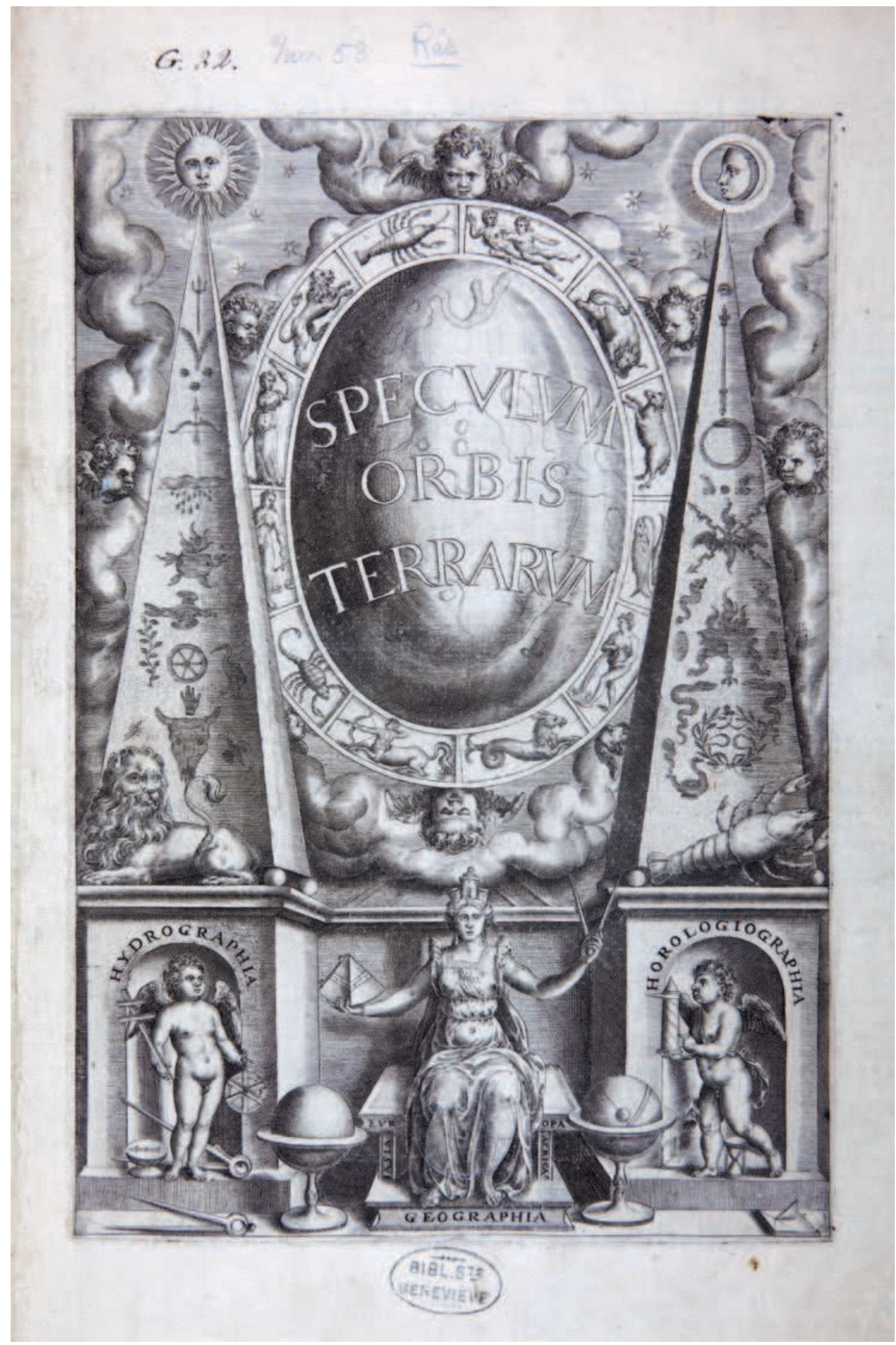

Page de titre du Speculum Orbis Terrarum de Gérard de Jode (1578 - coll. Bibliothèque Sainte-Geneviève) 


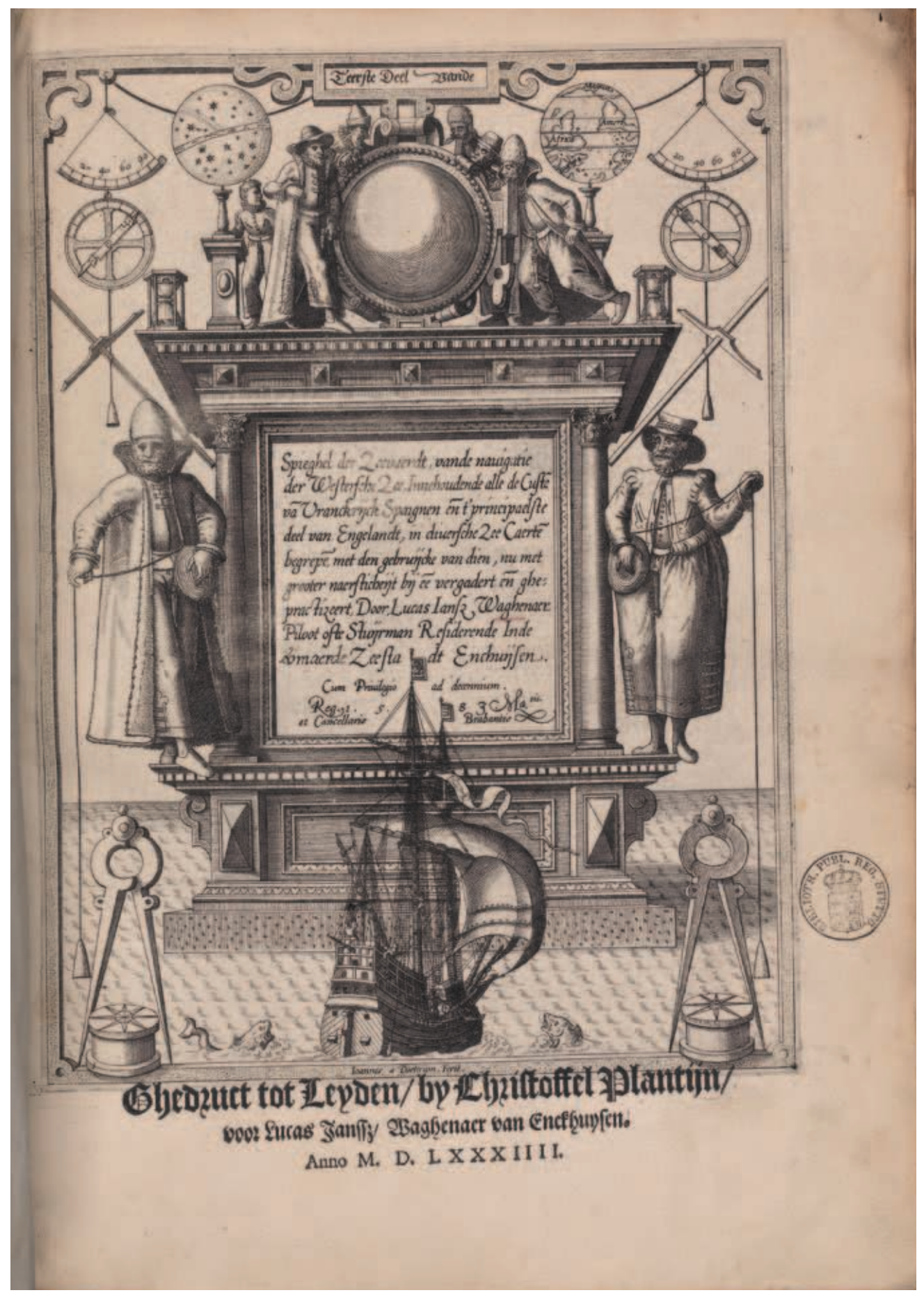

Page de titre du Spieghel der Zeevaert de Lucas Jeanszon Waghenaer (1584 - coll. Württembergische Landesbibliothek) 
tions qui permettent de tracer les cartes de la Terre. "Geographia " est d'ailleurs flanquée de deux globes, encore vierges de tout dessin. Le globe de droite est une sphère céleste comme l'indiquent le tracé de la ceinture du zodiaque et une toute petite sphère, qui paraît comme en suspension et qui représente sans doute la lune. La scène supérieure, en revanche, ouvre un espace cosmologique et figure le miroir, où les contours de la Terre apparaissent, visibles du spectateur de l'image, et non des trois personnages qui se trouvent à son pied. Ainsi figurée sur le frontispice du livre, l'efficacité symbolique du miroir apparaît comme dédoublée : exprimant la vocation encyclopédique et représentative du livre, il en est aussi l'image condensée, le symbole visuel. Le miroir est de ce point de vue une mise en abyme du frontispice lui-même, qui a pour vocation de résumer le livre qu'il introduit.

On retrouve le miroir géographique dans différents ouvrages d'instructions nautiques et de cartes marines, tel le Spieghel der Zeevaert ou Speculum Nauticum de Lucas Jeanszon Waghenaer, dans ses multiples éditions européennes ${ }^{24}$. La première impression de la première partie de l'ouvrage fut réalisée en 1583 par Plantin à Leyde. La nature même de ce livre justifie pleinement son titre. Comme le note excellemment D. Gernez, "le Spieghel der Zeevaert fut, à son époque, l'équivalent d'une petite bibliothèque pour gens de mer, contenant : un traité de cosmographie et de navigation, une table des marées, une sorte d'almanach nautique rudimentaire, un livre de directions de navigation pour les côtes de l'Europe septentrionale et occidentale et un certain nombre de cartes nautiques de ces rivages $"{ }^{25}$. Mais la page de titre prend à nouveau la métaphore du miroir au pied de la lettre.

Le frontispice a été gravé par Jan van Doetecum. Sur l'édition publiée à Leyde en 1586 , centrée autour du cartouche de titre, lui-même intégré dans un dispositif architectural, la scène s'organise en trois étages verticaux. Le fil conducteur qui les relie les uns aux autres est le fil à plomb : il souligne la dimension verticale de l'image et en même temps en indique le sens de lecture, la finalité. L'ensemble est bâti selon une symétrie rigoureuse entre la droite et la gauche, qui ne fait que renforcer les effets de centralité. L'étage supérieur est constitué par le miroir, offert de face au spectateur de l'image et vierge de tout tracé. Six personnages, de part et d'autre, regardent la surface miroitante. Les encadrant, deux globes : à gauche, une sphère céleste portant le dessin de quelques étoiles et de la ceinture du zodiaque sur laquelle sont suggérés quelques signes; sur la droite, nous avons un globe terrestre, divisé par les lignes des zones climatiques, et orienté sud en haut. On déchiffre des tracés géographiques, identifiés par les inscriptions "Africa ", " Europa ", " Ameri[ca] ", " Ter[ra] no[va] ", " Magellane ", cette dernière correspondant à la Terre des antipodes. Compas, sextants gradués, équerres et sabliers suggèrent les médiations techniques qui ont permis de construire ces artefacts comme de voyager sur les mers. Il y a un jeu évident entre la vision merveilleuse que partagent les six personnages autour du miroir pourtant aveugle, vision directe du reflet de la Terre et les substituts graphiques et tridimensionnels qui l'encadrent, où se trouvent figurés l'au-delà de la vision sensorielle, l'architecture graphique qui caractérise la carte : lignes départageant les zones climatiques, toponymes, bandeau zodiacal. L'étage intermédiaire figure deux personnages d'arpenteurs, perchés sur le bord de la corniche et laissant pendre deux fils à sonde jusqu'au niveau de la mer, où les attendent, de part et d'autre, le compas du navigateur et sa boussole. Un navire voguant toutes voiles déployées, entouré de deux monstres marins, rappelle la finalité de cet atlas maritime. Ce riche frontispice conduit donc de la vision magique dans le miroir ${ }^{26}$ à la navigation à l'estime, qui s'appuie sur les cartes, les directions et les distances. La multiplicité des instruments de mesure, la précision des gestes techniques et la présence même des deux globes, avec leurs tracés effectifs, montrent la transmission, en même temps que la validation du savoir et de la vision ${ }^{27}$. A l'évidence immédiate de la vision dans le miroir (dont est cependant exclu le spectateur de l'image) s'oppose l'expérience d'un pilote confirmé comme Lucas Jeanszon, qui multiplie les médiations techniques garantissant la valeur pratique comme l'exactitude mathématique des informations contenues dans le livre.

Le frontispice de l'édition d'Amsterdam, 1591, présentant des compléments et des descriptions dues à Richard Slotboom, a été regravé. S'il reprend la même architecture générale que le frontispice de 1586, il comporte cependant une variation notable : le miroir n'est plus entouré que de quatre personnes, dont deux enfants. Alors que le seul enfant de l'édition de 1586 se trouvait au pied du globe céleste et donc à la péri- 
phérie de la scène, sur l'édition de 1591, l'un d'eux se trouve agenouillé, le doigt pointé vers la surface brillante et tourné vers un adulte, penché, qui regarde aussi. Sur la gauche, c'est l'adulte qui a le doigt pointé vers la surface du globe, alors que le second enfant regarde. Est-ce un hasard si les rituels de la catoptromancie grecque décrits par A. Delatte mettent principalement en scène un enfant, jeune et pur, comme artisan principal de la vision magique ${ }^{28}$ ?

On notera en tout cas le changement de tonalité de cette série de frontispices par rapport au Speculum de de Jode et au Cosmographical Glasse de Cuningham. Les hiéroglyphes et l'imagerie zodiacale se sont effacés devant une profusion d'instruments de mesure. Le frontispice multiplie les signes de vérédiction à l'encontre des pilotes et gens de mer, destinataires de l'ouvrage.

Entre l'ouvrage de l'Anglais Cuningham et les atlas hollandais, il est un autre changement : le miroir passe en effet de la métaphore à la figuration. De métaphore désignant un genre livresque et un savoir encyclopédique, il devient un dispositif optique offrant une vision directe, sans médiations, sur l'espace terrestre. C'est sur des ouvrages hollandais que la métaphore du miroir apparaît prise au pied de la lettre, ou plutôt au pied de l'image, et ce n'est sans doute pas un hasard. Les frontispices de Gerard de Jode et de Waghenaer sont représentatifs de la nouvelle culture visuelle qui se développe aux Pays-Bas, aux $16^{\mathrm{e}}$ et $17^{\mathrm{e}}$ siècles, et qui a été si bien analysée par Svetlana Alpers ${ }^{29}$. Le miroir devient l'emblème même de la connaissance, une alternative au savoir apporté par les livres. Au côté des premiers télescopes et microscopes, ainsi que des chambres noires, le miroir est révélateur d'une nouvelle conception de la vision, du visible et de la représentation, et plus largement, le symbole d'un type de connaissance particulier, où l'esprit même devient le miroir de la nature ${ }^{30}$. Le miroir reste ainsi une métaphore récurrente pour le projet cartographique, au même titre que tous les verres optiques, qui élargissent les frontières du visible et du pensable ${ }^{31}$.

\section{Christian Jacob}

\section{Notes}

1 - Pour une présentation générale de cet imaginaire, voir J. Baltrusaitis, Essai sur une légende scientifique. Le miroir. Révelations, science-fiction et fallacies, Paris, Elmayan-Le Seuil, 1978

2 - H. Grabes (in The Mutable Glass. Mirror-imagery in titles and texts of the Middle Ages and English Renaissance, Cambridge, Cambridge university press [édition originale : Tübingen, 1973], 1982) explore la métaphore du miroir, particulièrement présente entre 1550 et 1650 , dans les titres de livres anglais du Moyen Age à la Renaissance.

3 - Grabes, op. cit., p. 40

4 - Patrologie latine, 172, col. 119-120

5 - Grabes, op. cit., p. 42

6 - Grabes, op. cit., p. 43

7- Grabes, op. cit., p. 44-48

8 - M. Corbett et R. W. Lightbown, The Comely Frontispice. The emblematic title-page in England 1550-1660, London, Henley and Boston, Routledge \& Kegan Paul, 1979, p. 34

9 - Corbett et Lightbown, op. cit., p. 35

10 - Cet ouvrage est reproduit en fac-similé par Da Capo Press, Theatrum Orbis Terrarum Ltd, Amsterdam et New York, 1968. Sur le contexte de l'œuvre de Cuningham et ses sources, voir E. G. R. Taylor, Tudor Geography 1458-1583, London, Methuen \& Co, 1930, p. 26-27. Pour une appréciation de sa place dans l'histoire de la géographie anglaise : D. Waters, The art of navigation in England in Elizabethan and early Stuart times, Greenwich, National Maritime Museum, $2^{\mathrm{e}}$ éd., 1978, vol. 1, p. 98-99.

11 - Sur ce frontispice, voir R. B. McKerrow et F. S. Ferguson, Title-page Borders used in England \& Scotland 1485-1640, London, printed for the bibliographical society at the Oxford University Press, 1932, planche 99 et notice p. 92-93 : le graveur est peut-être identifié par les initiales I B F dans l'angle inférieur gauche : Iohannes Bettes fecit (?)

12 - G. de Tervarent, Attributs et symboles dans l'art profane, 1450-1600. Dictionnaire d'un langage perdu, Genève, Droz, 1958, col. 198 et 403. McKerrow et Ferguson, op. cit., p. 93 suggèrent que ce Mercure barbu pourrait avoir les traits de John Day, l'imprimeur du livre.

13-McKerrow et Ferguson, op. cit., p. 92, interprètent ainsi cette zone emblématique : "Time bringing Truth and Antiquity to light ». Il est plus probable que le Temps soit entouré des trois stades de l'évolution humaine, l'enfant, l'adulte et le vieillard. Je remercie Ann Blair, Anthony Grafton et Marcia Reed qui m'ont aidé dans le déchiffrement de cette image.

14 - Comme me le suggère Marcia Reed (Getty Center, Special Collections).

15 - Tervarent, op. cit., col. 202, qui cite en particulier une gravure de Cornelis Massys.

16 - Op. cit., n. p. : «I [...] have devised this mirrour, or Cosmographical Glasse. In which men may behold not one or two personages, but the heavens with planets and starres, th'Earthe with her beautifull [sic] Regions, and the Seas with her merveilous increse ".

17 - Op. cit., fol. 120 : " So that observing this order of you prescribed, I may in like sorte at my pleasure, drawe a carde for Spaine, Fraunce, Germany, Italye, Graece, or any perticuler region: yea, in a warme \& pleasant house, without any perill of the raging Seas: danger of the enemies: losse of time: spending of substance: werines of body, or anguishe of minde. Oh how precious a Jewell is this, it may rightly be called a Cosmographicall Glasse, in which we may beholde the diversities of countries: natures of people \& innumerable formes of Beastes, Foules, Fishes, Trees, Frutes, Stremes, \& Meatalles ". 
18 - Nous avons consulté l'édition en fac-similé publiée par Theatrum Orbis Terrarum Ltd, Amsterdam, 1965, avec une introduction par R. A. Skelton. Pour la description de cet atlas, voir I. C. Koeman, Atlantes Neerlandici. Bibliography of terrestrial, maritime and celestial atlases and pilot books, published in the Netherlands up to 1880 , Amsterdam, Theatrum Orbis Terrarum, 1967-1985, vol. 2, p. 205-212. Pour une présentation générale de l'œuvre et de la biographie de Gérard de Jode et de son fils, voir F. Van Ortroy, L'œuvre cartographique de Gérard et de Corneille de Jode, Gand, Librairie scientifique E. Van Goethem \& Cie, 1914.

19 - On signalera en outre que les éditions abrégées du Theatrum réalisées par Pieter Heyns (1537-1597) et son fils Zacharias Heyns (1570-ca 1640) portent le titre de " Miroir du Monde » ou " Spieghel der Werelt ». L'une des raisons de ce passage du Theatrum au Speculum réside dans le projet éditorial de réaliser un abrégé de l'ouvrage d'Ortelius. L'épitomé de Pieter Heyns était même écrit en vers, ce qui contribua à son grand succès éditorial : Koeman, op. cit., vol. 2, p. 131-134.

20-Voir l'édition en fac-similé présentée par R. A. Skelton, G. de Jode, Speculum Orbis Terrarum, Antwerpen 1578, Amsterdam, Theatrum Orbis Terrarum Ltd, 1965

21 - D'autres frontispices sont construits sur ce modèle. Voir en particulier Nicolas Caussin, De Symbolica Aegyptiorum Sapientia, Paris, 1618 (reproduit dans E. Iversen, The Myth of Egypt and its Hieroglyphs in European Tradition, Copenhagen, Ged Gad Publishers, 1961, plate XVIII, fig. 2b)

22-En guise d'introduction à ce courant important de la symbolique de la Renaissance, voir R. Wittkower, Allegory and the Migration of Symbols, London, Thames and Hudson, 1987, p. 114-128, " Hieroglyphics in the early Renaissance "; Iversen, op. cit., p. 57-87; Corbett et Lightbown, op. cit., p. 22-25

23-Plotin, Ennéades, V.8.6

24-Voir le catalogue dans Koeman, op. cit., vol. 4 (1970), p. 465-501. Voir aussi I. C. Koeman, Miscellanea cartographica. Contributions to the history of cartography, edited by Günther Schilder and Peter van der Krogt, Utrecht, HES Publishers, 1988, p. 49-66 : « Lucas Janszoon Waghenaer: a sixteenth century marine cartographer " (reprint de l'article publié dans The Geographical Journal, 1965, 131, 2, p. 202-217)

25-D. Gernez, The Works of Lucas Janszoon Wagenaer, in The Mariner's Mirror, 1937, p. 336. Voir aussi Waters, op. cit., vol. 2, p. 168-175

26-Waters, op. cit., vol. 2, p. 169, commentant ce frontispice, indique que les marins regardent dans le miroir de la mer.

27-Le succès européen du Speculum explique peut-être l'influence de son frontispice : voir Koeman, 1967-1985, vol. 4, p. 469. Voir par exemple celui de Bartholomew Pitiscus, Trigonometry or the doctrine of triangles, 1614 (McKerrow et Ferguson, op. cit., 1932, n 265 et p. 200), où l'on retrouve des marins.

28-A. Delatte, La catoptromancie grecque et ses dérivés, Liège-Paris, Bibliothèque de la Faculté de Philosophie et Lettres de l'Université de Liège, 1932

29-S. Alpers, L'art de dépeindre. La peinture hollandaise au XVII siècle, traduit de l'anglais par Jacques Chavy, Paris, Gallimard, 1983

30-Alpers, op. cit., p. 67, 100

31 -Alpers, op. cit., p. 267,272 